PROCEEDINGS OF THE

AMERICAN MATHEMATICAL SOCIETY

Volume 138, Number 10, October 2010, Pages 3405-3413

S 0002-9939(2010)10373-8

Article electronically published on June 3, 2010

\title{
ARITHMETIC RIGIDITY
}

\author{
WAYNE RASKIND AND MATEI STROILA \\ (Communicated by Ted Chinburg)
}

\begin{abstract}
We prove an arithmetic analogue of rigidity results of Suslin and Beilinson, and then give some applications to countability of certain motivic cohomology groups of varieties over the complex numbers, assuming a finite generation of these groups for varieties over finitely generated fields.
\end{abstract}

\section{INTRODUCTION}

Let $K$ be a field and consider the Quillen $K$-group, $K_{3}(K)$. Denote by $K_{3}^{M}(K)$ the Milnor $K$-group of $K$ that is generated by symbols. The natural map

$$
K_{3}^{M}(K) \rightarrow K_{3}(K)
$$

is now known to be injective (it follows from results in $\mathrm{Su2}$ that the kernel is killed by 2). Let $K_{3}(K)^{\text {ind }}$ be the quotient of $K_{3}(K)$ by $K_{3}^{M}(K)$. This is called the indecomposable $K_{3}$. There is a regulator map, a real version of which was first considered by Borel [Bo] for number fields:

$$
K_{3}^{\text {ind }}(\mathbb{C}) \rightarrow \mathbb{C} /(2 \pi i)^{2} \mathbb{Z}
$$

Beilinson showed that the image of this regulator is countable by a rigidity argument (see $[\mathrm{Be}, 2.3 .4$ and $[\mathrm{M}], \S 3$, especially Corollary 3.6 ), which is what led to

Conjecture 1 (see e.g. [MS, Conjecture 11.5]). We have

$$
K_{3}^{\text {ind }}(\overline{\mathbb{Q}})=K_{3}^{\text {ind }}(\mathbb{C}),
$$

so that $K_{3}^{\text {ind }}(\mathbb{C})$ is a countable abelian group. Here $\overline{\mathbb{Q}}$ is the algebraic closure of $\mathbb{Q}$ in $\mathbb{C}$.

Unfortunately, it is not known whether the regulator map is injective.

There is an analogous situation for higher motivic cohomology. Let $X$ be a smooth projective variety over $\mathbb{C}$. Consider, for example, the motivic cohomology group $H^{3}(X, \mathbb{Z}(2))$, or, if you prefer, $H^{1}\left(X, \mathcal{K}_{2}\right)$, where $\mathcal{K}_{2}$ is the Zariski sheaf associated to the presheaf of Quillen $K_{2}$-groups. Using the identifications

$$
\operatorname{Pic}(X) \cong H^{2}(X, \mathbb{Z}(1)), \quad \mathbb{C}^{*} \cong H^{1}(X, \mathbb{Z}(1))
$$

and the product structure on motivic cohomology, one gets a product map:

$$
\operatorname{Pic}(X) \otimes \mathbb{C}^{*} \rightarrow H^{3}(X, \mathbb{Z}(2)) .
$$

Received by the editors November 19, 2008 and, in revised form, August 23, 2009.

2010 Mathematics Subject Classification. Primary 19F27, 19E15.

The first author was partially supported by NSA grant H98230-07-1-0041.

(C)2010 American Mathematical Society 
Let $H^{3}(X, \mathbb{Z}(2))^{\text {ind }}$ be the quotient of $H^{3}(X, \mathbb{Z}(2))$ by the image of the product map. Let $H^{i}\left(X, \mathbb{Z}_{\mathcal{D}}(j)\right)$ denote Deligne cohomology (see e.g. [EV]), and denote similarly $H^{3}\left(X, \mathbb{Z}_{\mathcal{D}}(2)\right)^{\text {ind }}$ as the quotient of $H^{3}\left(X, \mathbb{Z}_{\mathcal{D}}(2)\right)$ by the image of the product map:

$$
H^{1}\left(X, \mathbb{Z}_{\mathcal{D}}(1)\right) \otimes H^{2}\left(X, \mathbb{Z}_{\mathcal{D}}(1)\right) \rightarrow H^{3}\left(X, \mathbb{Z}_{\mathcal{D}}(2)\right) .
$$

Then Beilinson's rigidity result (loc. cit.) also applies to the image of the regulator (cycle class) map:

$$
H^{3}(X, \mathbb{Z}(2))^{\text {ind }} \rightarrow H^{3}\left(X, \mathbb{Z}_{\mathcal{D}}(2)\right)^{\text {ind }}
$$

and shows that this image is countable, which leads to:

Conjecture 2. For $X$ smooth and projective over any field $K$, the group $H^{3}(X, \mathbb{Z}(2))^{\text {ind }}$ is countable.

Briefly, such rigidity arguments are as follows: if $Y$ is a model of $X$ over an algebraic closure $K$ of some finitely generated field, then one shows using the proper base change theorem that the image of the regulator map all comes from the image of $H^{3}(Y, \mathbb{Z}(2))^{\text {ind }}$ and is therefore countable, since $K$ is countable. In this note, we give an arithmetic analogue of such rigidity results, hence the name, "arithmetic rigidity". As a consequence, we show that the images of suitable $\ell$-adic regulator maps are countable for indecomposable motivic cohomology groups on varieties over universal domains such as $\mathbb{C}$, and we show in some cases that if such motivic cohomology groups are finitely generated over any finitely generated ring over $\mathbb{Z}$, as is expected, then they are, in fact, countable over $\mathbb{C}$. At least in the case of indecomposable $K_{3}$, we strongly suspect that such results are known to some of the experts, but we could not find a reference. This follows easily from results of Suslin Su2, Merkur'ev-Suslin $\underline{\mathrm{MS}}$, and Levine Le. In fact, the rigidity lemma below is a fairly simple but very useful generalization of a result of Suslin ( $[\mathrm{Su} 2]$, Corollary 2.7).

This paper comprises part of the second author's Ph.D. thesis at the University of Southern California. The first author would like to thank B. Kahn, S. Lichtenbaum and A. Suslin for helpful conversations.

\section{Notation AND PRELIMINARIES}

Let $K$ be a field that is finitely generated over its prime subfield and let $L$ be a finitely generated, separable extension of $K$ in which $K$ is algebraically closed. We shall call such an $L$ a finitely generated regular extension of $K$. We denote by $\bar{K}$ a separable closure of $K$ and by $\bar{L}$ a separable closure of $L$.

Let $\ell$ be a prime number different from the characteristic of $K$. We denote by $\mathbb{Z} / \ell^{n} \mathbb{Z}(r)$ the group of $\ell^{n}$-th roots of unity, twisted $r$ times à la Tate, and $\mathbb{Z}_{\ell}(r)=\lim _{\leftarrow} \mathbb{Z} / \ell^{n} \mathbb{Z}(r)$. If $M$ is a finitely generated $\mathbb{Z}_{\ell}$ module with continuous action of $\operatorname{Gal}(\bar{K} / K)$, we set $M(r):=M \otimes_{\mathbb{Z}_{\ell}} \mathbb{Z}_{\ell}(r)$.

Let $X$ be a smooth projective variety over $K$. We denote by $\bar{X}$ the variety $X \times_{K} \bar{K}$. Consider the motivic cohomology groups $H^{i}(X, \mathbb{Z}(j))$. These may be taken in the sense of Bloch's higher Chow groups [Bl] or Voevodsky [V]. Then there are integral and rational cycle class maps:

$$
\begin{aligned}
& H^{i}(X, \mathbb{Z}(j)) \rightarrow H^{i}\left(X, \mathbb{Z}_{\ell}(j)\right), \\
& H^{i}(X, \mathbb{Q}(j)) \rightarrow H^{i}\left(X, \mathbb{Q}_{\ell}(j)\right) .
\end{aligned}
$$


Here the groups on the right are the continuous étale cohomology groups in the sense of Jannsen [J]. These are the right derived functors of the functor

$$
\left(\mathcal{F}_{n}\right) \mapsto \lim _{\leftarrow} H^{0}\left(X, \mathcal{F}_{n}\right)
$$

where $\left(\mathcal{F}_{n}\right)$ is an inverse system of sheaves of $\mathbb{Z} / \ell^{n} \mathbb{Z}$-modules on $X$.

Conjecture 3. The rational cycle class map is injective for $X$ as above.

There is a Hochschild-Serre spectral sequence:

$$
E_{2}^{r, s}=H^{r}\left(K, H^{s}\left(\bar{X}, \mathbb{Z}_{\ell}(j)\right)\right) \Longrightarrow H^{r+s}\left(X, \mathbb{Z}_{\ell}(j)\right) .
$$

We refer to the filtration on $H^{i}(X, \mathbb{Z}(j))$ that one gets by pulling back the filtration given by the spectral sequence as the Hochschild-Serre filtration.

From the spectral sequence, we get a map

$$
H^{i}(X, \mathbb{Z}(j))^{0} \rightarrow H^{1}\left(K, H^{i-1}\left(\bar{X}, \mathbb{Z}_{\ell}(j)\right)\right),
$$

where

$$
H^{i}(X, \mathbb{Z}(j))^{0}=\operatorname{ker}\left[H^{i}(X, \mathbb{Z}(j)) \rightarrow H^{i}\left(\bar{X}, \mathbb{Z}_{\ell}(j)\right)\right] .
$$

If $i \neq 2 j$, this last group is of finite index in $H^{i}(X, \mathbb{Z}(j))$, as follows easily from a specialization argument and the Weil conjectures as proved by Deligne (see [CTR1], Theorem 1 for this argument). Thus

$$
H^{i}(X, \mathbb{Q}(j))^{0}=H^{i}(X, \mathbb{Q}(j))
$$

if $i \neq 2 j$.

Lemma 1.1 (Rigidity Lemma). With notation as above, let $M$ be a finitely generated torsion free $\mathbb{Z}_{\ell}$-module with continuous action of $G=G a l(\bar{K} / K)$. Make $M$ into a $\mathcal{G}=\operatorname{Gal}(\bar{L} / L)$-module by making the kernel of the natural map $\mathcal{G} \rightarrow G$ act trivially. Assume:

(i) for all open subgroups $H$ of finite index in $G$, we have

$$
M(-1)^{H}=0
$$

and

(ii) for any abelian variety $A$ over $K$, we have that

$$
\left[T_{\ell}(A) \otimes_{\mathbb{Z}_{\ell}} M(-1)\right]^{G}=0,
$$

where $T_{\ell}(A)$ is the $\ell$-adic Tate module,

$$
\lim _{\leftarrow} A\left[\ell^{n}\right]
$$

Then the natural map

$$
H^{1}(K, M) \rightarrow H^{1}(L, M)
$$

is an isomorphism.

Remark 1.2. (i) Assumption (ii) of Lemma 1.1 is satisfied if $M$ is such that $V=M \otimes_{\mathbb{Z}_{\ell}} \mathbb{Q}_{\ell}$ is a Galois representation of pure weight different from -1 . To see this, let $A$ be an abelian variety over $K$ and consider

$$
T_{\ell}(A) \otimes_{\mathbb{Z}_{\ell}} M(-1) .
$$

By the Riemann hypothesis for abelian varieties as proved by Weil and a specialization argument, $T_{\ell}(A)$ is of pure weight -1 . Thus, if $M$ is of pure weight different from -1 , then $M(-1)$ is of pure weight different from 1 , 
and the tensor product above is of weight different from 0 , and hence has no $G$-invariants.

(ii) We shall need an analogous version of rigidity, where one takes an algebra $A$ that is finitely generated over the base field $K$. The proof is similar but easier than the case of a field $L$ that is finitely generated over $K$, and we omit it here.

Corollary 1. Let $\Omega$ be an uncountable algebraically closed field containing $K$. Then if $M$ satisfying the hypotheses of the lemma is a torsion free quotient of the étale cohomology group $H^{i-1}\left(\bar{X}, \mathbb{Z}_{\ell}(j)\right)$ with $i-1-2 j \neq-1,-2$, the image of the map

$$
H^{i}\left(X_{\Omega}, \mathbb{Z}(j)\right)^{0} \rightarrow \lim _{\left[K^{\prime}: \vec{K}\right]<\infty} \lim _{L \cdot \vec{g} \cdot / K^{\prime}} H^{1}(L, M)
$$

is countable. Here the outside limit is taken over all finite separable extensions of $K$ and the inside limit is taken over all finitely generated regular extensions of $K^{\prime}$.

Proof of the corollary assuming the Rigidity Lemma. Since we are dealing with $\ell$ adic cohomology for $\ell \neq \operatorname{char}(K)$, we reduce to the case where $\Omega$ is separably closed. We will use Remark 1.2 (ii) above. Let $K^{\prime}$ be a finite separable extension of $K$ and $L$ a finitely generated regular field extension of $K^{\prime}$. Any element of $H^{i}\left(X_{L}, \mathbb{Z}(j)\right)^{0}$ comes from an element of $H^{i}\left(X_{A}, \mathbb{Z}(j)\right)^{0}$, for some finitely generated $K^{\prime}$-algebra, $A$. Localizing, if necessary, we may assume that $A$ is regular, and replacing $K^{\prime}$ by a finite separable extension, if necessary, we may assume that the natural map $K^{\prime} \rightarrow A$ has a section. Consider the following obvious commutative diagram:

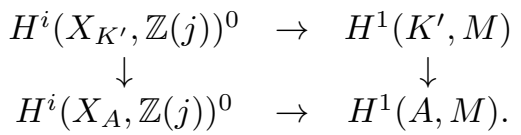

The vertical maps have splittings given by the section $A \rightarrow K^{\prime}$. From the diagram and the rigidity lemma, we see that the image of $H^{i}\left(X_{A}, \mathbb{Z}(j)\right)^{0}$ in $H^{1}(A, M)$ comes from the image of $H^{i}\left(X_{K^{\prime}}, \mathbb{Z}(j)\right)^{0}$ in $H^{1}\left(K^{\prime}, M\right)$. Note that this image is countable, since $K^{\prime}$ is finitely generated over the prime subfield. Taking the limit over all such finite extensions $K^{\prime}$ of $K$ and all regular finitely generated field extensions $L / K^{\prime}$, we see that the image of $(*)$ is a countable union of countable groups, so is countable, as claimed.

Example 1.3. (i) Let $L$ be a field that is finitely generated over $\mathbb{Q}$ and consider the motivic cohomology group $H^{1}(L, \mathbb{Z}(2))$. There is a regulator (cycle class) map

$$
H^{1}(L, \mathbb{Z}(2)) \rightarrow H^{1}\left(L, \mathbb{Z}_{\ell}(2)\right) .
$$

It is easy to see that the Galois module $\mathbb{Z}_{\ell}(2)$ satisfies the hypotheses of the rigidity lemma (in this case, the rigidity is due to Suslin (see [Su2], Corollary 2.7)). If $K$ is the algebraic closure of $\mathbb{Q}$ in $L$, then we get an isomorphism:

$$
H^{1}(K, M) \rightarrow H^{1}(L, M)
$$

(ii) Let $X$ be a smooth projective variety over $K$ and let $M=T_{\ell}(\operatorname{Br}(\bar{X}))(1)$, the $\ell$-Tate-module of the Brauer group of $\bar{X}$, twisted by one. Suppose that $T_{\ell}(\operatorname{Br}(\bar{X}))^{H}=0$ for all open subgroups $H$ of $G=\operatorname{Gal}(\bar{K} / K)$. Then assumption (i) of the lemma is satisfied. This is a consequence of the Tate conjecture for divisors for $X_{L}$ over every finite extension $L$ of $K$ together 
with semi-simplicity of the action of $G$ (if $K$ is a finite field or a number field, one can avoid assuming semi-simplicity). By Remark 1.2 (ii) above, assumption (ii) of the Rigidity Lemma is satisfied. Thus the Rigidity Lemma applies to such an $M$. Now it is not hard to see that there is a map

$$
H^{3}\left(X_{L}, \mathbb{Z}(2)\right)^{\text {ind }} \rightarrow H^{1}(L, M),
$$

so the corollary above applies. This applies to e.g. abelian varieties, K3 surfaces, etc.

Proof of the Rigidity Lemma. We have the inflation-restriction sequence,

$$
0 \rightarrow H^{1}(K, M) \rightarrow H^{1}(L, M) \rightarrow H^{1}(\bar{K} L, M)^{G} \rightarrow \cdots .
$$

Using the hypotheses, we show that the group on the right is zero. Our argument is actually very similar to that of Suslin ( $\underline{\mathrm{Su} 2}$, Corollary 2.7). Let $Y$ be a normal, projective, geometrically connected model of $L$ over $K$, and consider the exact sequence,

$$
0 \rightarrow \bar{K}(Y)^{*} / \bar{K}^{*} \rightarrow \operatorname{Div}(\bar{Y}) \rightarrow \operatorname{Pic}(\bar{Y}) \rightarrow 0 .
$$

Let $M_{n}=M / \ell^{n} M$ and recall the identification from Kummer theory:

$$
H^{1}\left(\bar{K} L, \mathbb{Z} / \ell^{n}(1)\right)=(\bar{K} L)^{*} /(\bar{K} L)^{* \ell^{n}} .
$$

Tensoring the sequence with $M_{n}(-1)$ and using the fact that $\operatorname{Div}(\bar{Y})$ is torsion free, we get the sequence

$$
\begin{gathered}
0 \rightarrow \operatorname{Tor}^{1}\left(\operatorname{Pic}(\bar{Y}), M_{n}(-1)\right) \rightarrow(\bar{K} L)^{*} \otimes M_{n}(-1) \\
\quad \stackrel{f_{n}}{\rightarrow} \operatorname{Div}(\bar{Y}) \otimes M_{n}(-1) \rightarrow \operatorname{Pic}(\bar{Y}) \otimes M_{n}(-1) .
\end{gathered}
$$

Taking projective limits and $G$-invariants, we get the exact sequence

$$
\begin{aligned}
0 & \rightarrow\left[\lim _{\leftarrow} \operatorname{Tor}^{1}\left(\operatorname{Pic}(\bar{Y}), M_{n}(-1)\right)\right]^{G} \rightarrow\left[\lim _{n}(\bar{K} L)^{*} \otimes M_{n}(-1)\right]^{G} \\
& \rightarrow\left[\lim _{n} \operatorname{Div}(\bar{Y}) \otimes M_{n}(-1)\right]^{G} .
\end{aligned}
$$

Note that all of the terms of the projective systems satisfy the Mittag-Leffler property, but since the groups in the system on the left are finite, we really don't need this to get the exactness of $(*)$.

We claim that the right and left terms of $(*)$ are zero using, respectively, hypotheses (i) and (ii). We deal with the right term first. For each irreducible codimension 1 subvariety $Z$ of $X$, let $k_{Z}$ be the algebraic closure of $K$ in the function field of $Z$ and let $H_{Z}$ be the absolute Galois group of $K_{Z}$. Let $W_{Z}$ be an irreducible subvariety of $\bar{Y}$ lying over $Z$. Then, by Shapiro's lemma and hypothesis (i), we have

$$
\lim _{\leftarrow}\left(\operatorname{Div}(\bar{Y}) \otimes M_{n}(-1)\right)^{G} \subseteq \prod_{Z}\left(\bigoplus_{W \mapsto Z} M(-1)_{W}\right)^{G}=\prod_{Z} \bigoplus_{W_{Z}} M(-1)^{H_{Z}}=0 .
$$

As for the left term of the exact sequence $(*)$, an easy computation shows that

$$
\left(\underset{\leftarrow}{\lim _{n}} \operatorname{Tor}^{1}\left(\operatorname{Pic}(\bar{Y}), M_{n}(-1)\right)\right)^{G} \cong\left(T_{\ell}(\operatorname{Pic}(\bar{Y})) \otimes M(-1)\right)^{G}=0,
$$

by assumption (ii). This completes the proof of the Rigidity Lemma. 


\section{Countability}

Let $K$ be a field that is finitely generated over $\mathbb{Q}$. There is a finitely generated $\mathbb{Q}$-algebra $A$ that is regular and has fraction field $K$. Let $Y=\operatorname{Spec}(A)$. Then there is an exact sequence of motivic cohomology groups,

$$
\cdots H^{1}(Y, \mathbb{Z}(2)) \rightarrow H^{1}(K, \mathbb{Z}(2)) \rightarrow \lim _{\vec{Z}} H_{Z}^{2}(Y, \mathbb{Z}(2)) \rightarrow \cdots
$$

where the limit is taken over all codimension one subschemes $Z$ of $Y$. Standard conjectures on the finite generation of motivic cohomology groups of schemes finitely generated over $\mathbb{Z}$ would imply that the left group is finitely generated. An easy induction argument using purity and the vanishing of $H^{0}(W, \mathbb{Z}(1))$ for $W$ smooth shows that the right group is zero. Thus we expect that $H^{1}(K, \mathbb{Z}(2))$ is finitely generated, although we have no idea how to prove this at present. Note that $H^{1}\left(K, \mathbb{Z}(2) \cong K_{3}^{\text {ind }}(K)\right.$.

Recall a theorem of Levine ([Le], Theorem 4.12) and of Merkur'ev-Suslin ([MS $)$ that says there is an isomorphism

$$
\lim _{\leftarrow} K_{3}(K)^{i n d} / \ell^{n} \rightarrow H^{1}\left(K, \mathbb{Z}_{\ell}(2)\right)
$$

Theorem 2.1. Assume that for every field $K$ that is finitely generated over $\mathbb{Q}$, $H^{1}(K, \mathbb{Z}(2))$ is a finitely generated abelian group. Then the group $H^{1}(\mathbb{C}, \mathbb{Z}(2))$ is countable.

Proof. We have that $H^{1}(\mathbb{C}, \mathbb{Z}(2))=\lim _{[K: \overrightarrow{\mathbb{Q}}]<\infty} \lim _{L f . g . / K} H^{1}(L, \mathbb{Z}(2))$, where the limit is taken over all finite extensions $K / \mathbb{Q}$ and all finitely generated regular field extensions $L$ of $K$. From the theorem of Levine and Merkur'ev-Suslin just mentioned and the assumption of finite generation of $H^{1}(L, \mathbb{Z}(2))$, we have that the Chern class map

$$
H^{1}(L, \mathbb{Z}(2)) \rightarrow H^{1}\left(L, \mathbb{Z}_{\ell}(2)\right)
$$

has kernel that is torsion prime to $\ell$. By the rigidity lemma and its corollary, the image of the injective map

$$
H^{1}(\mathbb{C}, \mathbb{Q}(2)) \rightarrow \lim _{[K: \overrightarrow{\mathbb{Q}}]<\infty} \lim _{L f \cdot \vec{g} \cdot / K} H^{1}\left(K, \mathbb{Q}_{\ell}(2)\right)
$$

is countable, and hence the group on the left is countable. Finally, one knows that the torsion of $H^{1}(\mathbb{C}, \mathbb{Z}(2))$ is countable by Suslin's rigidity theorem [Su1] (also proved by Gabber and Gillet-Thomason [GT]). This completes the proof of the theorem.

Remark 2.2. The rigidity part of this result was proved by Suslin Su2], who also noticed the remark about finite generation implying countability. Since this last part was not in the literature, we included it here.

Before stating the next result, we define $H^{3}(X, \mathbb{Q}(2))^{\text {ind }}$ for $X$ smooth and projective over a field $K$. Consider the product map

$$
\left[\operatorname{Pic}(X) \otimes_{\mathbb{Z}} \mathbb{Q}\right] \otimes_{\mathbb{Q}}\left[K^{*} \otimes_{\mathbb{Z}} \mathbb{Q}\right] \rightarrow H^{3}(X, \mathbb{Q}(2))
$$

(here we use the identifications $\operatorname{Pic}(X)=H^{2}(X, \mathbb{Z}(1)), K^{*}=H^{1}(X, \mathbb{Z}(1))$ ).

Let $H^{3}(X, \mathbb{Q}(2))^{\text {dec }}$ be the image of this map. Then we define $H^{3}(X, \mathbb{Q}(2))^{\text {ind }}$ to be the quotient $H^{3}(X, \mathbb{Q}(2)) / H^{3}(X, \mathbb{Q}(2))^{\text {dec }}$. If $C$ is a curve over $K$, we have that $H^{3}(C, \mathbb{Q}(2))^{\text {ind }}=0$, as follows easily from the Gersten-Quillen complex which 
computes the cohomology of $\mathcal{K}_{2}$ (note that we have $H^{3}(C, \mathbb{Z}(2))=H^{1}\left(C, \mathcal{K}_{2}\right)$ ). If $K$ is algebraically closed, we can define $H^{3}(X, \mathbb{Z}(2))^{\text {ind }}$ in a similar way. To define $H^{3}(X, \mathbb{Z}(2))^{\text {ind }}$ when $K$ is not algebraically closed is not difficult but is a bit tedious, as we have to take norms from finite extensions of $K$. However, this is not necessary for what we want to prove here.

Theorem 2.3. Let $X$ be a smooth projective, geometrically connected variety over C. Assume that

(i) for any smooth proper model $\mathcal{X}$ of $X$ over a ring $A$ that is finitely field over $\mathbb{Z}$, the group $H^{3}(\mathcal{X}, \mathbb{Z}(2))$ is finitely generated (as is expected),

(ii) the Tate conjecture for divisors is true for any model $Y$ of $X$ over a field $K$ that is finitely generated over $\mathbb{Q}$, and the absolute Galois group of $K$ acts semi-simply on $H^{2}\left(Y_{\bar{K}}, \mathbb{Q}_{\ell}(1)\right)$.

Then $H^{3}(X, \mathbb{Z}(2))^{\text {ind }}$ is countable.

Proof. The proof is very similar to the proof of Theorem 2.1, and we only sketch it here. Let $Y$ and $K$ be as in the statement of the theorem. Let $\mathcal{Y}$ be a smooth proper model of $Y$ over a suitable ring $A$ that is finitely generated over $\mathbb{Z}$ with fraction field $K$. Then the injection (see e.g. Su2], Corollary 4.4)

$$
H^{3}(Y, \mathbb{Z}(2)) / \ell^{n} \rightarrow H_{e t}^{3}\left(Y, \mathbb{Z} / \ell^{n}(2)\right)
$$

and the assumption of finite generation of $H^{3}(\mathcal{Y}, \mathbb{Z}(2))$ give us an injection

$$
H^{3}(Y, \mathbb{Q}(2))^{i n d} \rightarrow H^{3}\left(Y, \mathbb{Q}_{\ell}(2)\right)^{i n d},
$$

where the group on the right is the quotient of $H^{3}\left(Y, \mathbb{Q}_{\ell}(2)\right)$ by the image of the product map

$$
H^{1}\left(Y, \mathbb{Q}_{\ell}(1)\right) \times H^{2}\left(Y, \mathbb{Q}_{\ell}(1)\right) \rightarrow H^{3}\left(Y, \mathbb{Q}_{\ell}(2)\right) .
$$

Let $\bar{Y}=Y \times_{K} \bar{K}$, let $\operatorname{Br}(\bar{Y})$ be the Brauer group of $\bar{Y}$, and let

$$
V_{\ell} B r(\bar{Y})=\left(\lim _{\leftarrow} B r(\bar{Y})\left[\ell^{n}\right]\right) \otimes_{\mathbb{Z}_{\ell}} \mathbb{Q}_{\ell}
$$

be its $\ell$-adic Tate vector space. Note that for any algebraically closed field $M$ containing $\bar{K}$, we have

$$
V_{\ell} B r(\bar{Y}) \cong V_{\ell} B r\left(Y_{M}\right) .
$$

Consider the exact sequence

$$
0 \rightarrow N S(\bar{Y}) \otimes \mathbb{Q}_{\ell}(1) \rightarrow H^{2}\left(\bar{Y}, \mathbb{Q}_{\ell}(2)\right) \rightarrow V_{\ell} B r(\bar{Y})(1) \rightarrow 0
$$

that comes from taking cohomology of the Kummer exact sequence of sheaves on $Y$ and Tate-twisting by 1 . Then for any field $L$ that is finitely generated over $K$, we have a map

$$
H^{3}\left(Y_{L}, \mathbb{Q}(2)\right)^{i n d} \rightarrow H^{1}\left(L, V_{\ell} B r(\bar{Y})(1)\right) .
$$

The hypotheses ensure that the rigidity lemma applies to the $\operatorname{Gal}(\bar{K} / K))$-representation $V_{\ell} \operatorname{Br}(\bar{Y})(1)$ (see Example 1.3 (ii)), and so by Corollary 1, the image of the map

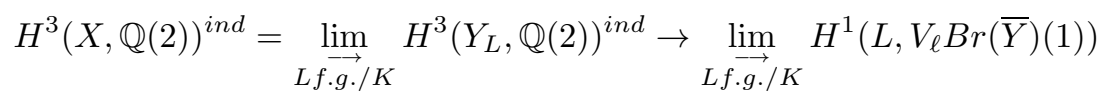

is countable. We claim that the other graded quotients of $H^{3}(X, \mathbb{Q}(2))^{\text {ind }}$ for the Hochschild-Serre filtration (see $§ 1$ ) do not give any additional contribution. To see 
this for $F^{2}$, take a generic curve $C$ on $Y$, e.g. by taking the (complete) $(d-1)$ fold intersection of smooth hyperplane sections, where $d=\operatorname{dim}(Y)$. Consider the diagram

$$
\begin{array}{cccc}
F^{2} H^{3}(Y, \mathbb{Q}(2)) & \rightarrow & H^{2}\left(K, H^{1}\left(\bar{Y}, \mathbb{Q}_{\ell}(2)\right)\right) \\
\downarrow & & \downarrow \\
F^{2} H^{3}(C, \mathbb{Q}(2)) & \rightarrow & H^{2}\left(K, H^{1}\left(\bar{C}, \mathbb{Q}_{\ell}(2)\right)\right) .
\end{array}
$$

By the weak Lefschetz theorem and Poincaré complete reducibility (see CTR2, $\S 4$ for this argument), the right vertical arrow is injective. But $H^{3}(C, \mathbb{Q}(2))^{\text {ind }}=0$ by the remark preceding the statement of this theorem, and hence the top horizontal arrow is zero after passing to the indecomposable quotients. This proves the claim for $F^{2}$. As for $F^{3}$, an argument similar to that in ( $\underline{\mathbb{R}}$, Proposition 2.2) shows that $F^{3}=F^{3+j}$ for any $j \geq 0$. By hypothesis, the filtration is separated, and hence $F^{3}=0$. Thus we have an injection

$$
H^{3}(Y, \mathbb{Q}(2))^{\text {ind }} \rightarrow H^{1}\left(K, V_{\ell}(B r(\bar{Y})(1)) .\right.
$$

Since the group on the left is countable and the image of these maps as $Y$ ranges over models of $X$ over finitely generated fields is rigid, we see that $H^{3}(X, \mathbb{Q}(2))^{\text {ind }}$ is countable. Now by ([CTR1, Theorem 2.1) the torsion of $H^{3}(X, \mathbb{Z}(2))$ is countable, and this completes the proof of the theorem.

\section{REFERENCES}

[Be] A.A. Beilinson, Higher regulators and values of L-functions, J. of Soviet Math. 30 (1985) 2036-2070. MR760999 (86h:11103)

[Bl] S. Bloch, Algebraic cycles and higher K-theory, Advances in Math. 61 (1986) 267-304. MR852815 (88f:18010)

[Bo] A. Borel, Stable real cohomology of arithmetic groups, Annales Sci. École Normale Supérieure 7 (1974) 235-272. MR0387496 (52:8338)

[CTR1] J.-L. Colliot-Thélène and W. Raskind, $\mathcal{K}_{2}$-cohomology and the second Chow group, Math. Annalen 270 (1985) 165-199. MR771978 (86m:14005)

[CTR2] J.-L. Colliot-Thélène and W. Raskind, Groupe de Chow de codimension deux des variétés algébriques sur les corps de nombres: un théorème de finitude pour la torsion, Inventiones Math. 105 (1991) 241-265. MR.1115542 (92j:14009)

[EV] H. Esnault and E. Viehweg, Deligne-Beŭlinson cohomology. Beĭlinson's conjectures on special values of L-functions, 43-91, Perspect. Math., 4, Academic Press, Boston, MA, 1988. MR 944991 (89k:14008)

[GT] H. Gillet and R.W. Thomason, On the K-theory of strictly Henselian local rings and a theorem of Suslin, J. Pure and Applied Algebra 34 (1984) 241-254. MR772059(86e:18014)

[J] U. Jannsen, Continuous étale cohomology, Math. Annalen 280 (1988) 207-245. MR929536 (89a:14022)

[Le] M. Levine, The indecomposable $K_{3}$ of fields, Annales Sci. École Normale Supérieure 22 (1989) 255-344. MR1005161 (91a:11061)

[MS] A.S. Merkur'ev and A. Suslin, The group $K_{3}$ for a field, Izv. Akad. Nauk. SSSR 54 (1990) 339-356, English translation in Mathematics of the USSR: Izvestia 36 (1990) 541-565. MR1072694 (91g:19002)

[Mi] J.S. Milne, Étale Cohomology, Princeton Mathematical Series, Volume 33, Princeton University Press, 1980. MR559531 (81j:14002)

[M] S. Müller-Stach, Constructing indecomposable motivic cohomology classes on algebraic surfaces, J. Algebraic Geometry 6 (1997) 513-543. MR.1487225 (99k:14016)

[R] W. Raskind, Higher -adic Abel-Jacobi mappings and filtrations on Chow groups, Duke J. Math. 78 (1995) 33-57. MR1328751 (96d:14009)

[Su1] A.A.Suslin, On the K-theory of algebraically closed fields, Inventiones Math. (1983) 241245. MR714090 (85h:18008a) 
[Su2] A. A. Suslin, Homology of $\mathrm{GL}_{n}$, characteristic classes and Milnor K-theory. (Russian) Algebraic geometry and its applications. Trudy Mat. Inst. Steklov. 165 (1984), 188-204, English translation in Algebraic $K$-theory, number theory, geometry and analysis (Bielefeld, 1982), 357-375, Lecture Notes in Math., 1046, Springer, Berlin, 1984. MR752941 (86f:11090a); MR752941 (86f:11090b)

[Su3] A.A. Suslin, Torsion in $K_{2}$ of fields, J. of K-Theory 1 (1987) 1-29. MR899915 (89a:11123)

[V] V. Voevodsky, Triangulated categories of motives over a field, in Cycles, Transfers and Motivic Homology Theories, E. Friedlander, A. Suslin and V. Voevodsky, editors, Annals of Mathematics Studies, Volume 143, Princeton University Press, 1999. MR.1764202

School of Mathematical and Statistical Sciences, Arizona State University, P.O. Box 871804, Tempe, Arizona 85287-1804

E-mail address: raskind@asu.edu

Emerging Technologies, NAVteq, 425 West Randolph Street, Chicago, Illinois 60606

E-mail address: matei.stroila@navteq.com 